Background The field of anaesthesiology is multidisciplinary and includes the perioperative trajectory, but also the domains of pain, palliative, intensive and emergency care. In The Netherlands there are numerous guidelines on anaesthesiology, from generic to disease and target group specific. These were developed by different stakeholders and were not always thoroughly checked on consistency with other guidelines.

Objective To assess uniformity in recommendations in the field of anesthesiology.

Methods Four guidelines were considered the base of anaesthesiology care; pre-, peri- and postoperative care and postoperative pain treatment. The recommendations of these four guidelines were combined with a number of consensus statements and matched with disease and target group specific recommendations. These recommendations were categorised into three groups: 1) no controversy; 2) controversy, update necessary; 3) new guideline(s) needed. For the recommendations in the categories 2 and 3 a working group was formed to address these issues.

Results The inventory is on-going and will be finished in spring 2013.

Discussion The total number of guidelines and recommendations on the topic of anaesthesiology are great. This makes it complex for the clinician to find the right recommendation and calls for a more convenient way of presenting them. Supervision from the anaesthesiology association is required for the development of new guidelines to guarantee uniformity between anaesthesiology recommendations.

Implications for Guideline Developers/Users It is of great importance that recommendations throughout guidelines are never conflicting. An electronic modular database could be a more convenient way of presenting recommendations.

\section{P118 ARE LEVELS OF EVIDENCE FROM DIFFERENT CLINICAL PRACTICE GUIDELINES COMPARABLE? - TESTING OF A METHOD FOR STANDARDIZATION OF DIFFERENT EVIDENCE GRADING SYSTEMS}

W Hoffmann-Eßer, R Großelfinger, N Holzmann, C Brockhaus, S Ein Waldt, C Ernsting, A Yurdakul, A Rüther, U Siering. Institute for Quality and Efficiency in Health Care (IQWiG), Cologne, Germany

\section{0:1136/bmjqs-2013-002293.170}

Background In 2009 we presented a method for standardisation of different evidence grading systems (EGS) to simplify the comparison of levels of evidence (LoE) from different guidelines. For this purpose, LoE from guidelines were allocated to a reference standard, the EGS from the Federal Joint Committee's (G-BA) Code of Procedure. This approach has not yet been tested on several different EGS from guidelines.

Objective To test the feasibility of a method for standardisation of different EGS from COPD, asthma and breast cancer guidelines.

Methods We conducted a systematic search for the above guidelines in guideline databases and websites of guideline providers. The search period covered 11/2007 to 7/2012. Eligible guidelines were evidence-based English or German guidelines using an EGS. The LoE reported were allocated to the EGS from the G-BA's Code of Procedure.

Results 43 guidelines on chronic diseases with 19 different EGS and 188 different LoE were included. With 4 exceptions, all LoE used in the EGS could be allocated to at least one category of the reference standard. In 44 cases, the LoE from the identified EGS could be allocated to exactly one category and in 63, an LoE was allocated to several categories. Several LoE from one guideline were allocated to one category in 15 cases; this can result in loss of information.

Discussion The testing of a method for standardisation of different EGS indicates that standardisation of LoE using a reference standard can be successfully implemented and can simplify the comparison of different EGS.

\section{P119 THE RARE-BEST PRACTICES PROJECT: AN OVERVIEW}

${ }^{1} \mathrm{D}$ Taruscio, ${ }^{1} \mathrm{C}$ Morciano, ${ }^{1} \mathrm{P}$ Laricchiuta, ${ }^{1} \mathrm{G}$ Vincenti, ${ }^{1} \mathrm{~L}$ Agresta, ${ }^{2} \mathrm{P}$ Mincarone, ${ }^{2} \mathrm{~F}$ Palazzo, ${ }^{2} \mathrm{C}$ Leo, ${ }^{2} \mathrm{~S}$ Sabina, ${ }^{2} \mathrm{R}$ Guarino, ${ }^{3} \mathrm{~J}$ Auld, ${ }^{4} \mathrm{~T}$ Sejersen. 'Istituto Superiore di Sanità, Rome, Italy; ${ }^{2}$ Consiglio Nazionale delle Ricerche, Rome, Italy; ${ }^{3}$ Jamarau, London, UK; ${ }^{4}$ Karolinska Institutet, Stockholm, Sweden

\section{0:1136/bmjqs-2013-002293.171}

Background RARE-Best Practices is a 4-year project (20132016) funded by the EU FP7.

Objective Developing a sustainable networking platform, supporting an efficient exchange of reliable and up to date information on the management of rare diseases (RD) to improve patient health outcomes.

Methods RARE-Best Practices will reach its goals by promoting collaboration among partners with a strong track record in RD research as well as in clinical practice guidelines (CPG) and systematic review development from academic institutions, governmental bodies, patients organisations and networks.

Results Project expected outputs: 1) identification of challenges to be considered in deriving high quality standards for CPG on $\mathrm{RD}$; 2) creation of transparent procedures and criteria for the evaluation and the collection of CPG on RD in a publicly searchable database; 3) identification of the available notations for graphic representation of processes within CPG to improve user understandability and implementation; 4) production of mechanisms to identify and prioritise RD clinical research needs to optimise the research agenda on $\mathrm{RD} ; 5$ ) development of training activities targeted to key stakeholders to disseminate process and tools for developing and evaluating CPG.

Discussion/Implication for Guidelines Developers Users RAREBest Practices will address the patients and health care providers demand for updated and high quality CPG on RD. It intends also to respond to the Directive 2011/24/EU which encourages EU MS to the development of European Reference Networks in the area of $\mathrm{RD}$ which, among other criteria and conditions, 'should have the capacity to produce good practice guidelines'.

\section{P121 CLINICAL DECISION SUPPORT: A VALUABLE TOOL FOR MANY REASONS}

T Van Vegchel, S Kersten, M Harmsen. Comprehensive Cancer Centre The Netherlands, Utrecht, The Netherlands

\section{0:1136/bmjqs-2013-002293.172}

Background Oncology is complex and time-consuming care. Because evidence changes frequently, implementation of knowledge is viable for putting evidence into daily practice and decreasing variation in treatment advice.

Context Clinical Decision Support (CDS) based on Clinical Practice Guidelines improves both individual care for cancer patients, including increase in safety, efficiency and transparency and 